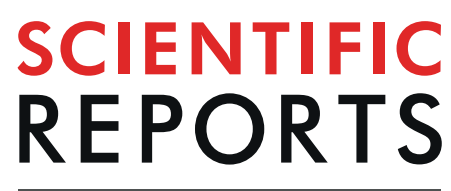

natureresearch

\title{
Apelin/APJ signaling suppresses the pressure ulcer formation in cutaneous ischemia-reperfusion injury mouse model
}

\author{
SahoriYamazaki ${ }^{1}$, Akiko Sekiguchi ${ }^{1}$, Akihiko Uchiyama ${ }^{1}$, Chisako Fujiwara ${ }^{1}$, Yuta Inoue ${ }^{1}$, \\ Yoko Yokoyama ${ }^{1}$, Sachiko Ogino ${ }^{1}$, Ryoko Torii ${ }^{1}$, Mari Hosoi ${ }^{1}$, Ryoko Akai ${ }^{2}$, Takao Iwawaki ${ }^{2}$, \\ Osamu Ishikawa ${ }^{1} \&$ Sei-ichiro Motegi ${ }^{1 *}{ }^{*}$
}

Several studies have demonstrated potential roles for apelin/APJ signaling in the regulation of oxidative stress associated with ischemia-reperfusion (I/R) injury in several organs. Objective was to assess the role of apelin/APJ signaling in the development of pressure ulcers (PUs) formation after cutaneous I/R injury in mice. We identified that cutaneous I/R injury increased the expression of apelin in the skin at I/R site. Administration of apelin significantly inhibited the formation of PUs. The reductions of blood vessels, hypoxic area and apoptosis in I/R site were inhibited by apelin injection. Oxidative stress signals in OKD48 mice and the expressions of oxidative stress related genes in the skin were suppressed by apelin injection. $\mathrm{H}_{2} \mathrm{O}_{2}$-induced intracellular $\mathrm{ROS}$ and apoptosis in endothelial cells and fibroblasts were suppressed by apelin in vitro. Furthermore, MM07, biased agonist of APJ, also significantly suppressed the development of PUs after cutaneous I/R, and the inhibitory effect of MM07 on PUs formation was higher than that in apelin. We conclude that apelin/APJ signaling may inhibit cutaneous I/R injuryinduced PUs formation by protecting the reduction of vascularity and tissue damage via suppression of oxidative stress. Exogenous application of apelin or MM07 might have therapeutic potentials against the development of PUs.

Pressure ulcers (PUs) are one of the common skin diseases which usually occur elder people and patients with perceptual or movement disorder. In addition, the patients with PUs sometimes cause fatal outcome by local and systemic infections. There has long been considered that the pathogenesis of PUs was associated with tissue damage caused by external force and ischemia. However, there has been increasing evidence that cutaneous ischemia-reperfusion (I/R) is important in the pathogenesis of PUs ${ }^{1-3}$. I/R injury is defined as cellular injury caused by the reperfusion of blood to previously ischemic tissue ${ }^{4,5}$. It is described that reperfusion of blood into the hypoxic tissue triggers off adverse events, including thrombosis and capillary narrowing which lead to vasculopathy, infiltration of inflammatory cells, production of proinflammatory cytokines and the apoptosis of resident cells and necrosis of tissues ${ }^{6}$. I/R injury causes multiple diseases, such as vascular infarction or spasm of brain, heart, kidney and skin. Reactive oxygen species (ROS), such as $\mathrm{H}_{2} \mathrm{O}_{2}$ and $\mathrm{NO}$, are also known as a key player that exacerbate the tissues damage caused by $\mathrm{I} / \mathrm{R}$ injury ${ }^{7}$.

Apelin, an endogenous ligand of G protein-coulpled receptor APJ (putative receptor protein related to the angiotensin receptor AT1 $)^{8,9}$ has multiple functions in the regulation of cardiovascular hemostasis, angiogenesis and adipose tissue function via apelin/APJ signaling ${ }^{10-13}$. APJ is expressed ubiquitously, especially in endothelial cells and vascular smooth muscle cells $8,9,11,12$. Furthermore, recent studies demonstrated that apelin/APJ signaling prevented oxidative stress, resulting in the inhibition of diabetic microvascular complications ${ }^{14,15}$ and the protection of tissue damage caused by I/R injury in kidney, heart and brain ${ }^{16-18}$. However, it has not been elucidated whether apelin/APJ signaling regulates cutaneous I/R injury associated with PUs. Herein, we analyzed the role and mechanisms of the regulation of cutaneous I/R-induced PUs formation by apelin/APJ signaling.

${ }^{1}$ Department of Dermatology, Gunma University Graduate School of Medicine, Maebashi, Japan. ${ }^{2}$ Division of Cell Medicine, Department of Life Science, Medical Research Institute, Kanazawa Medical University, Ishikawa, Japan.

*email: smotegi@gunma-u.ac.jp 
A

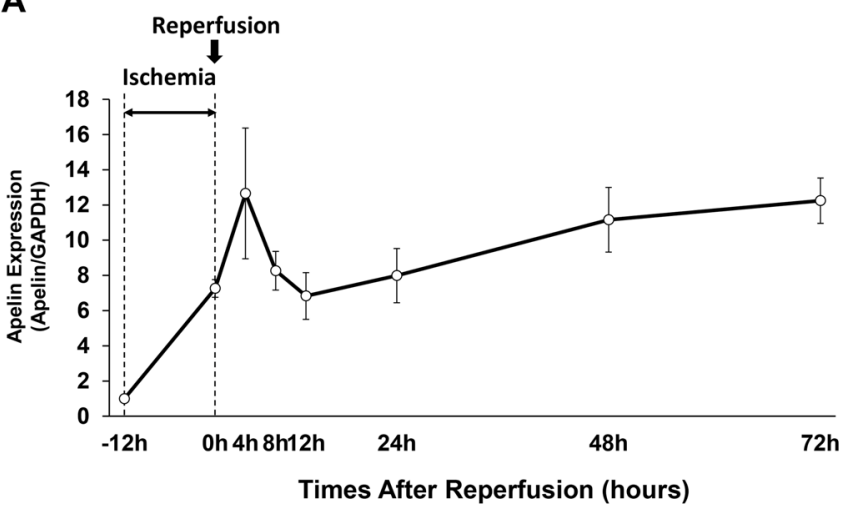

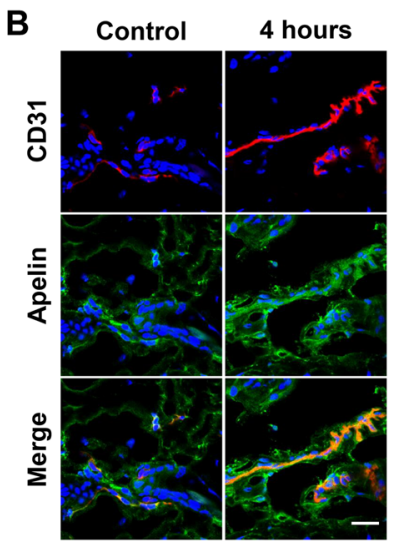

Figure 1. Apelin expression and distribution during ischemia-reperfusion (I/R) injury. (A) Quantification of apelin mRNA levels in the I/R site from the beginning of ischemia to 72 hours after reperfusion by quantitative reverse transcriptase-PCR (RT-PCR). The end of ischemia was assigned 0 hour. Data are relative to mRNA level in -12 hours. Values were determined in $n=4$. (B) Expression and distribution of apelin in the skin before ischemia (control) and at 4 hours after reperfusion (red: CD31, green: apelin). Scale bar $=20 \mu \mathrm{m}$. All values represent mean \pm SEM. $* * P<0.01, * P<0.05$.

\section{Results \\ Expression of apelin during cutaneous I/R in vivo. First, we investigated the apelin expression during cutaneous I/R injury. mRNA levels of apelin expression in the skin of I/R site were increased after ischemia $(0 \mathrm{~h})$ and 4 hours after reperfusion (Fig. 1A). Then, apelin expression was immediately decreased from 4 to 12 hours after reperfusion. After that, it was gradually increased following 1 to 3 days after I/R injury (Fig. 1A). To examine the amount and distribution of apelin under normal and after reperfusion condition in murine skin, immuno- fluorescence staining was performed. Apelin was accumulated around CD31+ endothelial cells in the dermis in the peripheral area of I/R site, and the expression of apelin was increased after reperfusion (Fig. 1B). These results suggest that apelin expression might be increased by cutaneous I/R-induced hypoxia, and apelin may be mainly produced by $I / R$ affected endothelial cells in the peripheral area of $I / R$ site.}

Apelin protected PUs formation after cutaneous I/R in mice model in vivo. To assess the effect of apelin ([Pyr $\left.{ }^{1}\right]$-Apelin-13) on the development of PUs after cutaneous I/R in vivo, we compared wound area after $\mathrm{I} / \mathrm{R}$ injury in normal C57BL/6 mice treated with subcutaneous injection of apelin or PBS as a control. We used a simple, reproducible and noninvasive experimental mouse model to evaluate the pathogenesis of cutaneous PUs by I/R in vivo ${ }^{3,19}$. Administration of apelin significantly inhibited the formation of PUs (Fig. 2A,B). The wound area in apelin-injected mice was significantly smaller that in control mice from 1 to 5 days after reperfusion. At 4 days after reperfusion, the size of wound area in the apelin-injected mice was $70 \%$ of that in the control mice. Next, we examined the expression of apelin in the wound healing process in I/R mice with or without apelin treatment, and found that administration of apelin did not change the mRNA levels of apelin in I/R site at 4, 12 and 48 hours after cutaneous I/R injury (Fig. 2C). These results demonstrate that apelin partially protected the formation of cutaneous PUs after cutaneous I/R.

Injection of apelin protected vascular loss by cutaneous I/R injury. We previously identified that the number of blood vessels was reduced after cutaneous I/R injury in mice ${ }^{20}$. Therefore, we investigated the effect of apelin on vascular loss caused by cutaneous I/R injury. At 4 days after reperfusion, the numbers of CD31 ${ }^{+}$ endothelial cells and $\mathrm{NG}^{+}$pericytes around I/R areas in apelin-treated mice were significantly higher than those in control mice (Fig. 3A). These results suggest that apelin might prevent the reduction of vascularity after cutaneous I/R injury.

Apelin suppressed hypoxia and apoptosis by cutaneous I/R injury. We next examined the influence of apelin treatment on tissue hypoxia after cutaneous I/R injury in mice. Hypoxic area in the skin tissue was analyzed using the hypoxia marker pimonidazole. At one day after cutaneous I/R, hypoxic area in the I/R site was increased compared with control mice without I/R (Fig. 3B). However, hypoxia staining positive area in I/R site in apelin-treated mice were significantly decreased compared to those in vehicle-treated mice (Fig. 3B). As hypoxia activates cellular apoptosis ${ }^{21}$, we examined the influence of apelin on the number of apoptotic cells in I/R areas in mice. TUNEL staining revealed that administration of apelin into I/R site reduced the increased number of apoptotic cells after I/R injury (Fig. 3C). These results suggest that administration of apelin might suppress hypoxic area and apoptosis in I/R site after cutaneous I/R injury.

Apelin regulated the infiltration of inflammatory cells and the expressions of cytokines and growth factors after cutaneous I/R. We previously reported that cutaneous I/R injury induced the infiltration of inflammatory cells in I/R site, and the growth factors and cytokines produced by inflammatory cells were key factors following the development of PUs and wound healing process ${ }^{22,23}$. To examine the effect of apelin 
A

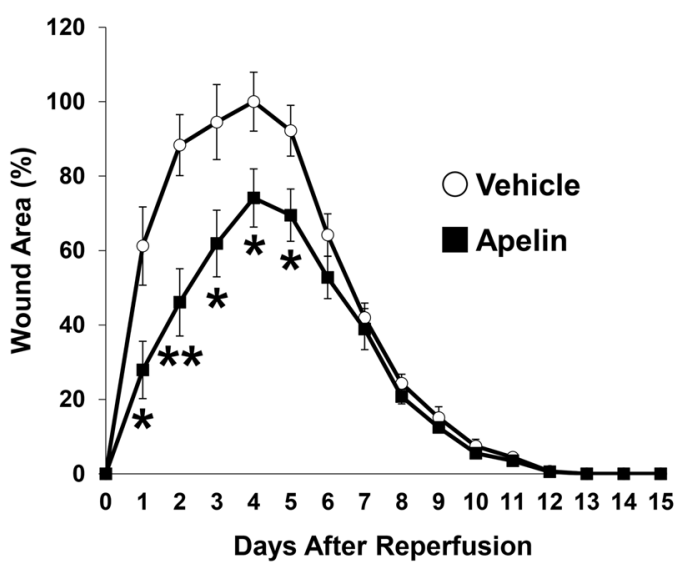

B

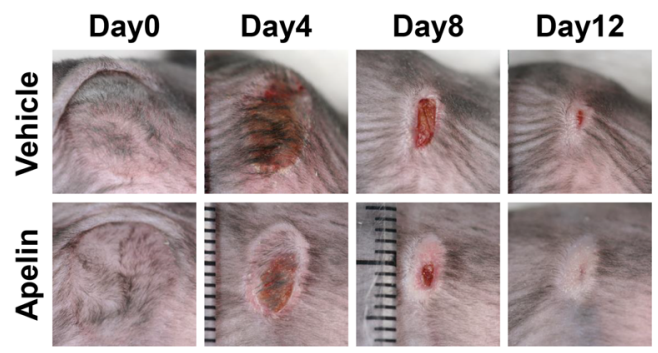

C

4h

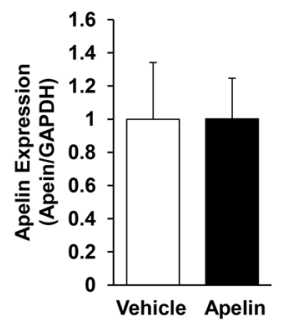

$12 \mathrm{~h}$

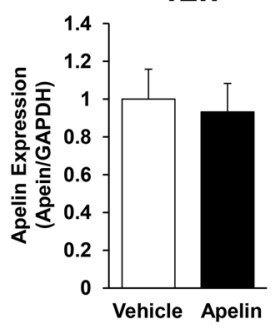

$48 \mathrm{~h}$

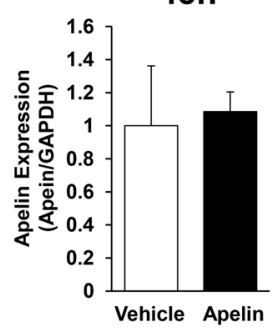

Figure 2. Apelin protected PUs formation in cutaneous I/R injury mouse model. (A) The size of the wound area after I/R injury in normal C57BL/6 mice treated with subcutaneous injection of apelin $(10 \mathrm{ng} / \mathrm{mice})$ or phosphatebuffered saline as a control. The size of the ulcer in control mice at 4 days after reperfusion was assigned a value of $100 \%$ (vehicle: $\mathrm{n}=9$, apelin: $\mathrm{n}=10$, for each time point and group). (B) Representative images of the wound after cutaneous $\mathrm{I} / \mathrm{R}$ in control or apelin treated mice at $0,4,8$, and 12 days after reperfusion. (C) Comparison of mRNA levels of apelin expression during wound healing in I/R site between control and apelin-treated mice at 4, 12 and 48 hours after I/R injury. $\mathrm{n}=5$. All values represent mean \pm SEM. $* * P<0.01, * P<0.05$.

administration on the dynamics of infiltration of inflammatory cells $\left(\mathrm{MPO}^{+}\right.$neutrophils, $\mathrm{CD}^{+} 8^{+}$macrophages and $\mathrm{CD}^{+} \mathrm{T}$ cells), immunohistochemical studies were performed using skin tissue of I/R site between control and apelin-treated mice at day 0, 1, 4 and 7 after I/R injury. At day 1, the number of $\mathrm{MPO}^{+}$neutrophils was significantly inhibited by apelin treatment (Fig. 4A). However, there were no significant difference between control and apelin-treated mice at day 0,4 and 7 . The numbers of $\mathrm{CD}_{6} 8^{+}$macrophages and $\mathrm{CD}^{+} \mathrm{T}$ cells at day 1 was significantly inhibited by apelin, and the peak of the numbers of infiltrated $\mathrm{CD} 68^{+}$macrophages and $\mathrm{CD} 3^{+} \mathrm{T}$ cells were delayed by apelin treatment (Fig. 4A).

Furthermore, we examined the expression levels of growth factors and cytokines expression, including tumor necrosis factor- $\alpha$ (TNF- $\alpha$ ), transforming growth factor- $\beta$ (TGF- $\beta$ ), interleulin- 6 (IL-6), IL-10 and basic fibroblast growth factor (bFGF) in I/R site through wound healing process by real-time PCR assay. The expression levels of proinflammatory cytokines (TNF- $\alpha$, IL-6) were not different between control and apelin-treated mice through day 0 to 7 . The mRNA levels of IL-10 and bFGF expressions were significantly increased at day 1 by apelin treatment, and TGF- $\beta$ expressions was significantly increased at day 1 to 4 by apelin treatment (Fig. 4B). These results suggest that apelin might suppress recruitment of inflammatory cells at early phase of wound healing, and change the expressions of several growth factors and cytokines in the I/R site.

Apelin suppressed oxidative stress after cutaneous I/R. Next, we investigated the effect of apelin on oxidative stress induced by I/R injury by using OKD48 (Keap1-dependent oxidative stress detector, NO-48) mice $^{24}$. OKD48 mice have a transgene encoding a modified Nuclear factor erythroid 2-related factor 2 (Nrf2), 
A
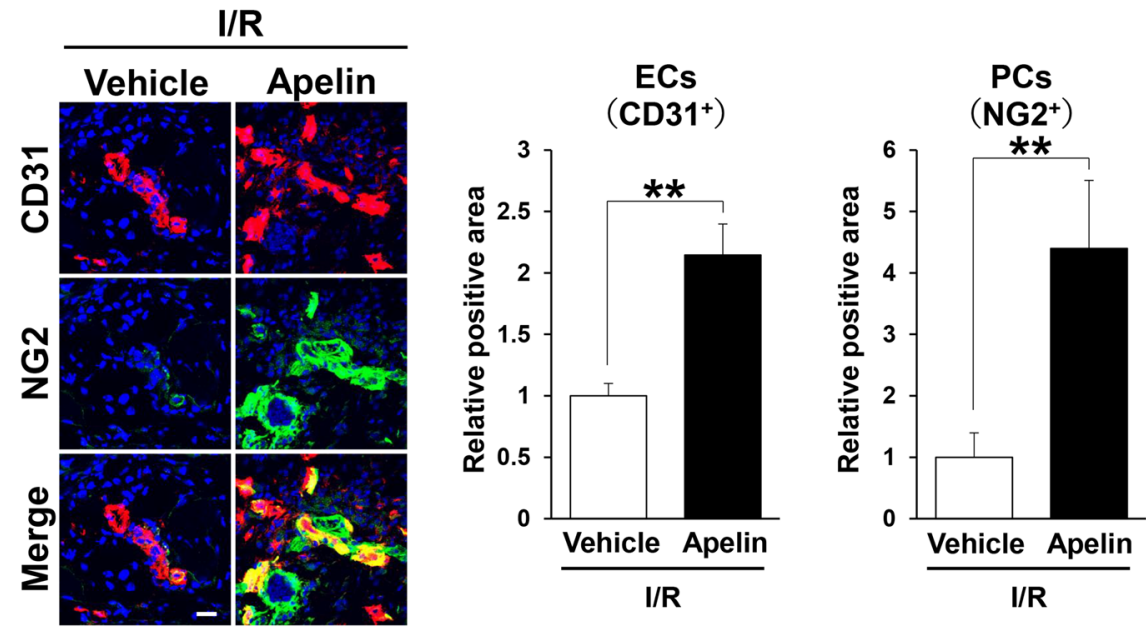

B
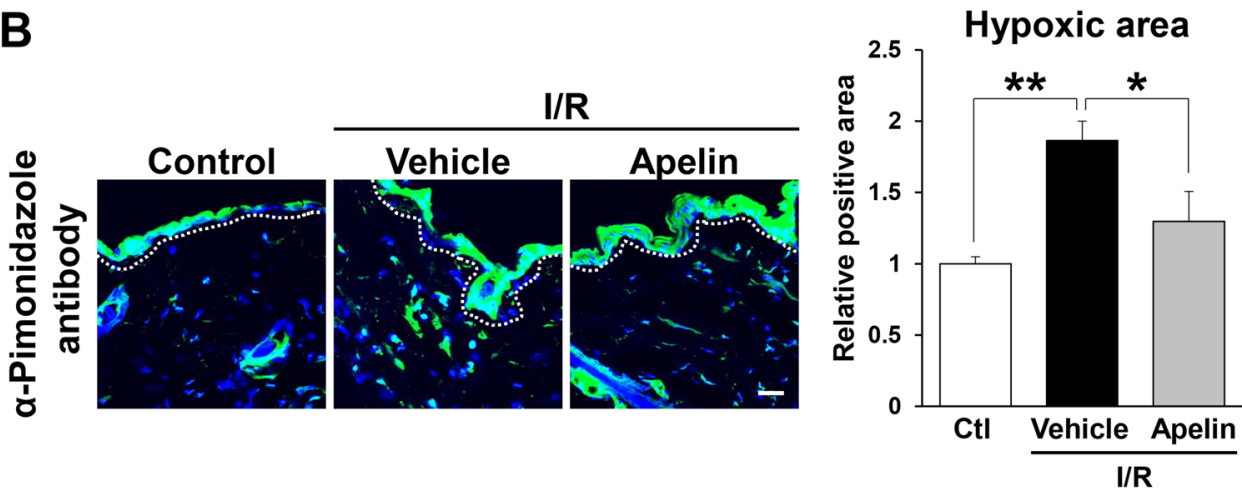

C

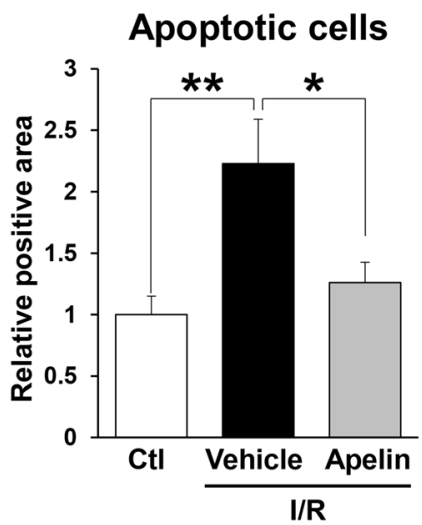

Figure 3. Apelin protected vascular reduction and suppressed hypoxia and apoptosis after cutaneous I/R. (A) The amount of $\mathrm{CD} 31^{+}$ECs and NG2 ${ }^{+}$pericytes in the cutaneous I/R area at 4 days after reperfusion. (B) The amount of pimonidazole ${ }^{+}$hypoxic area in cutaneous $\mathrm{I} / \mathrm{R}$ site at 1 day after reperfusion. Quantification of the pimonidazole $^{+}$areas in 6 random microscopic fields from the center of $I / R$ area in $n=3$ mice per groups was performed using ImageJ software. Positive area in control mice was assigned a value of 1 . Scale bar $=20 \mu \mathrm{m}$. (C) The number of apoptotic cells in the I/R site at 1 day after reperfusion was determined by counting both TUNEL- and DAPI-positive cells. Values were determined in 6 random microscopic fields in $\mathrm{n}=3$ mice per group. All values represent mean \pm SEM. $* * P<0.01, * P<0.05$.

which is an essential transcription factor for expression of antioxidative stress genes ${ }^{25}$. By using this mouse strain, oxidative stress in vivo can be detected with luminescence signals ${ }^{20,24,26}$. At 1 day after reperfusion, luminescence signal was significantly enhanced in the I/R area, and this signal was drastically reduced by administration of apelin (Fig. 5A,B). Additionally, the mRNA expression levels of oxidative stress-related factors, including Nrf2, heme oxygenase 1 (HO-1) and thioredoxin-2 (Trx2) in the I/R area were examined using real-time PCR. It has been reported that I/R injury enhances the expression of Nrf2, Trx 2 and HO-1 in the brain and liver ${ }^{27-29}$. Consistent with previous results, mRNA levels of Nrf2, HO-1 and Trx2 expressions were significantly upregulated after 
A

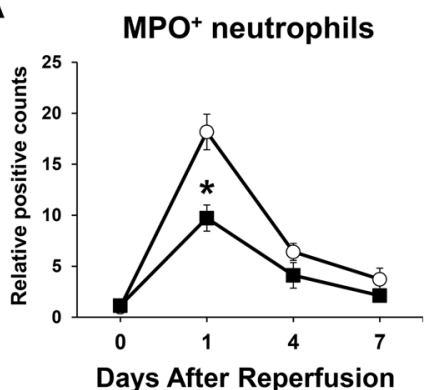

B
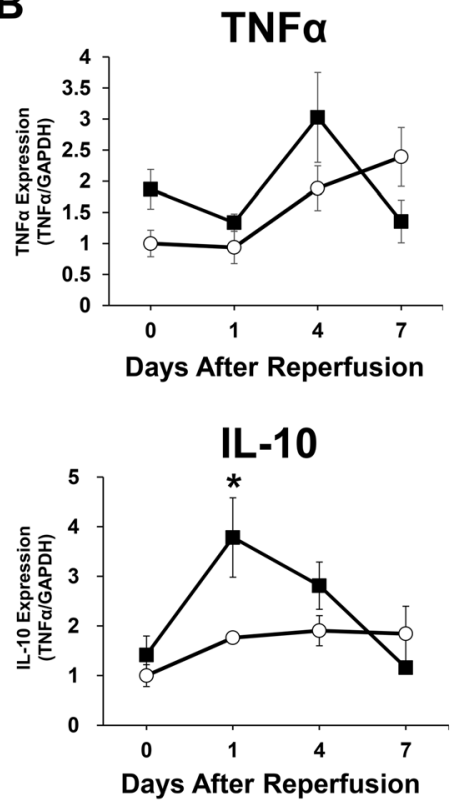

$\mathrm{CD}^{+}{ }^{+}$macrophages

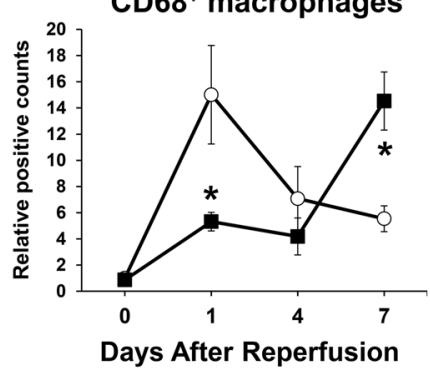

IL-6

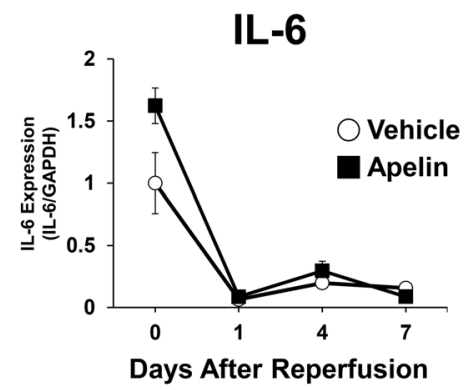

bFGF

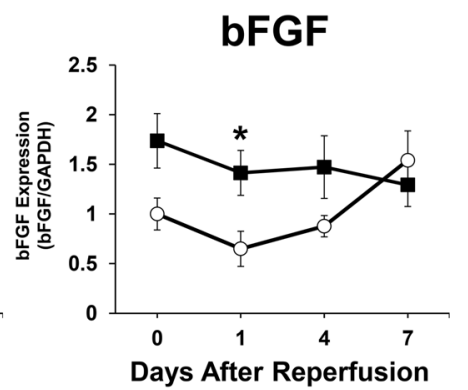

$\mathrm{CD}^{+} \mathrm{T}$ cells

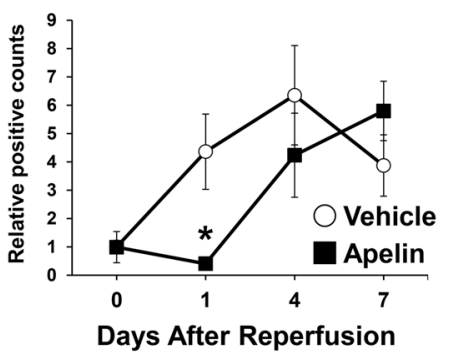

Days After Reperfusion

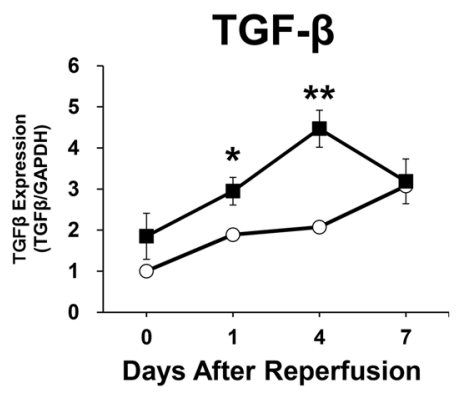

Figure 4. Apelin regulated the infiltration of inflammatory cells and the expressions of cytokines and growth factors after cutaneous I/R. (A) Quantification of the numbers of infiltrating $\mathrm{MPO}^{+}$neutrophils, $\mathrm{CD}^{+} 8^{+}$ macrophages and $\mathrm{CD}^{+} \mathrm{T}$ cells in I/R site at $0,1,4$ and 7 days after I/R treated with or without apelin. Values were determined by counting cells in six random microscopic fields in $n=4-5$ mice per groups. (B) mRNA levels of growth factors and cytokines in I/R site at 0, 1, 4 and 7 days after I/R treated with or without apelin. $\mathrm{n}=4-5$ mice in each group. The number of cells or mRNA levels in control mice were assigned values of 1 . All values represent mean \pm SEM. $* * P<0.01, * P<0.05$.

cutaneous I/R injury, and apelin injection suppressed those gene expressions (Fig. 5C). These results suggest that the oxidative stress in cutaneous I/R area might be inhibited by apelin injection.

Apelin suppressed ROS production and apoptosis driven by oxidative stress in vitro. Next, we examined whether apelin suppress ROS production and oxidative stress-induced apoptosis of endothelial cells and fibroblasts in vitro. $\mathrm{H}_{2} \mathrm{O}_{2}$-induced ROS productions in endothelial cells was suppressed by apelin treatment (Fig. 6A). In addition, apelin treatment significantly reduced the amount of $\mathrm{H}_{2} \mathrm{O}_{2}$-induced early apoptotic and necrotic cells in fibroblasts (Fig. 6B). These results suggest that apelin might reduce oxidative stress and oxidative stress-induced cell apoptosis in vitro.

MM07 protected PUs formation after cutaneous I/R injury in vivo. Finally, we investigated the effect of the synthetic biased agonist of APJ, MM0 $7^{30,31}$, on the development PUs formation after I/R injury in vivo. Since it has been reported that MM07 has greater potential than apelin to increase the forearm blood flow in human ${ }^{30}$, the amount of MM07 for treatment was designed $1 / 10$ with respect to treatment with apelin (Fig. $2 ; 10 \mu \mathrm{g}$ apelin, Fig. 7; $1 \mu \mathrm{g}$ MM07). Wound area after I/R injury in C57BL/6 mice treated with subcutaneous injection of MM07 or PBS as a control were analyzed. Administration of MM07 significantly inhibited the formation of PUs after cutaneous I/R injury (Fig. 7A,B). At 4 days after reperfusion, the size of the wound area in the MM07-injected mice was $50 \%$ of that in the control mice. These results suggest that MM07 might have protective effects on the development of PUs formation after cutaneous I/R injury, and that MM07 might have greater potential than apelin to inhibit PUs formation in vivo. 
A
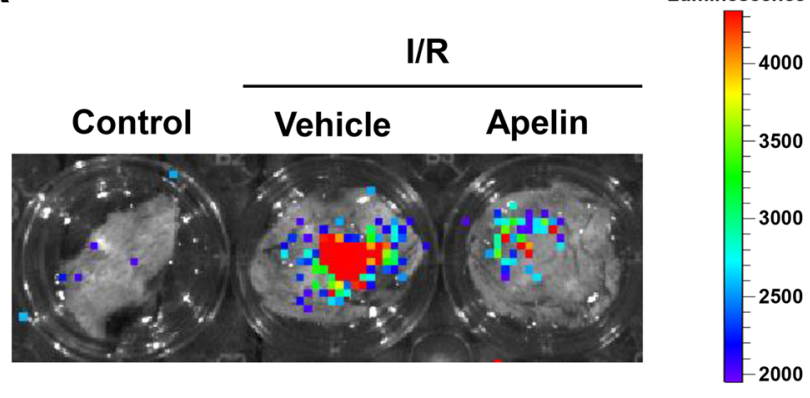

$\mathrm{p} / \mathrm{sec} / \mathrm{cm}^{2} / \mathrm{sr}$

Color bar

Min $=1950$

$\operatorname{Max}=4340$

C

Nrf2

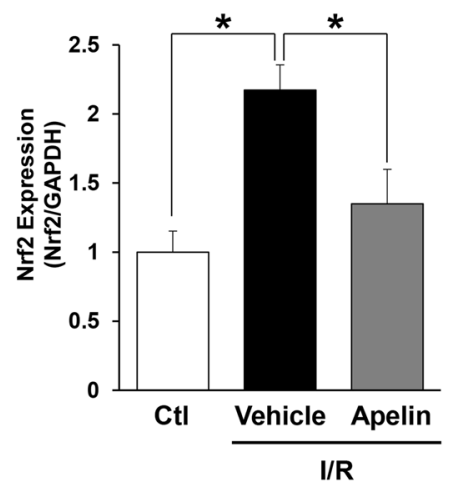

Trx2

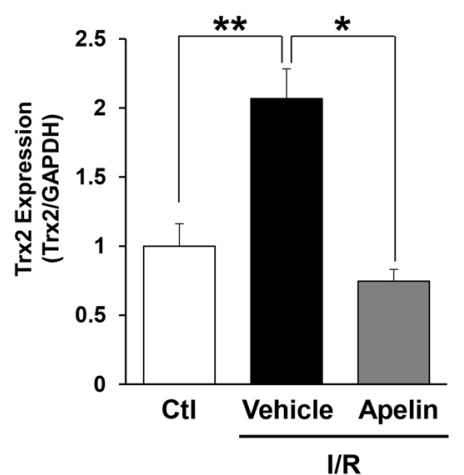

B

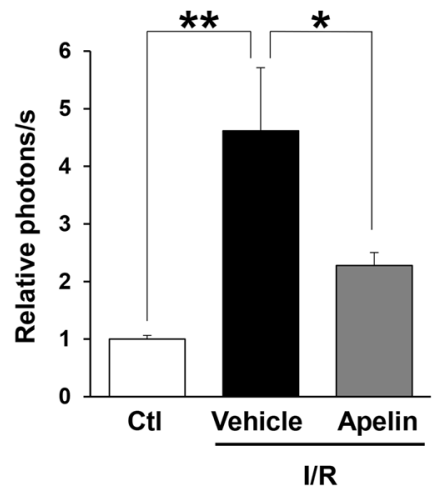

HO-1

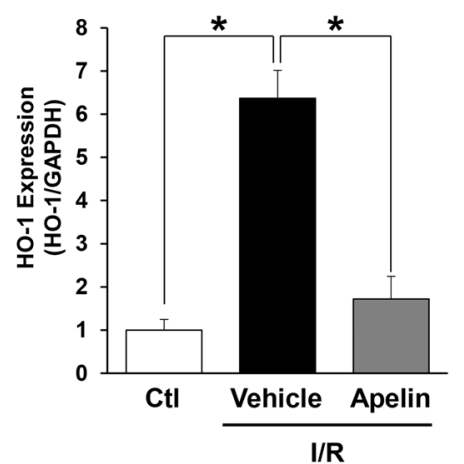

Figure 5. Apelin reduced oxidative stress induced by cutaneous I/R injury in vivo. (A) Representative image of luminescence signals in cutaneous I/R area in OKD48 mice at 24 hours after reperfusion (Day 1). The color scale bar shows the photon counts (photon $(\mathrm{p}) / \mathrm{sec} / \mathrm{cm}^{2} / \mathrm{sr}$ ). (B) Quantification of luminescence signals in cutaneous $\mathrm{I} / \mathrm{R}$ area in OKD 48 mice. $\mathrm{n}=4$ in each group. (C) mRNA levels of oxidative stress-associated factors, Nrf2, Trx2 and $\mathrm{HO}-1$ in the I/R area at 24 hours after reperfusion (Day 1). mRNA levels in control mice were assigned as values of 1 . Values represent mean \pm SEM. $\mathrm{n}=4-5$ mice in each group. $* * P<0.01, * P<0.05$.

A

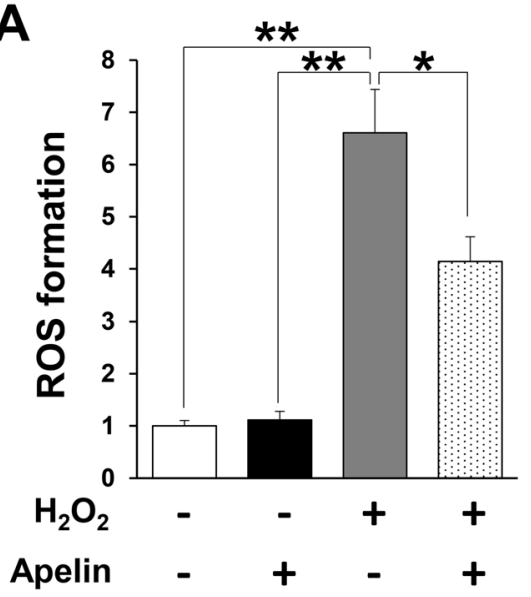

B

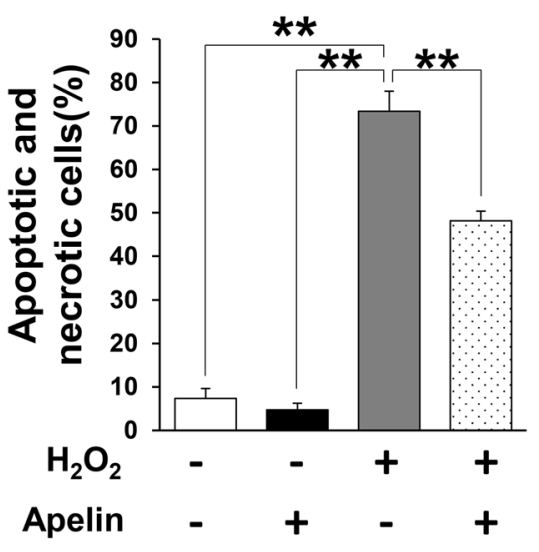

Figure 6. Apelin inhibited the oxidant-induced intracellular accumulation of ROS in endothelial cells and apoptosis in fibroblasts in vitro. (A) Quantification of $\mathrm{H}_{2} \mathrm{O}_{2}$-induced $(0.75 \mathrm{mM})$ intracellular ROS production in HUVECs treated with or without apelin. ROS formation in cells without treatment was assigned as a value of 1 . $\mathrm{n}=4$ in each group. (B) Representative data of the amount of early apoptotic cells (Annexin $\mathrm{V}^{+}, 7-\mathrm{AAD}^{-}$) and necrotic cells (Annexin $\mathrm{V}^{+}, 7-\mathrm{AAD}^{+}$) in NIH3T3 fibroblasts with or without $\mathrm{H}_{2} \mathrm{O}_{2}(0.75 \mathrm{mM})$ and/or apelin. (C) Quantitation of the amount of early apoptotic cells (Annexin $\mathrm{V}^{+}, 7-\mathrm{AAD}^{-}$) and necrotic cells (Annexin $\mathrm{V}^{+}$, 7-AAD $\left.{ }^{+}\right)$in NIH3T3 fibroblasts with or without $\mathrm{H}_{2} \mathrm{O}_{2}(0.75 \mathrm{mM})$ and/or apelin. Values represent means $\pm \mathrm{SEM}$ in four independent experiments. $* * P<0.01, * P<0.05$. 
A

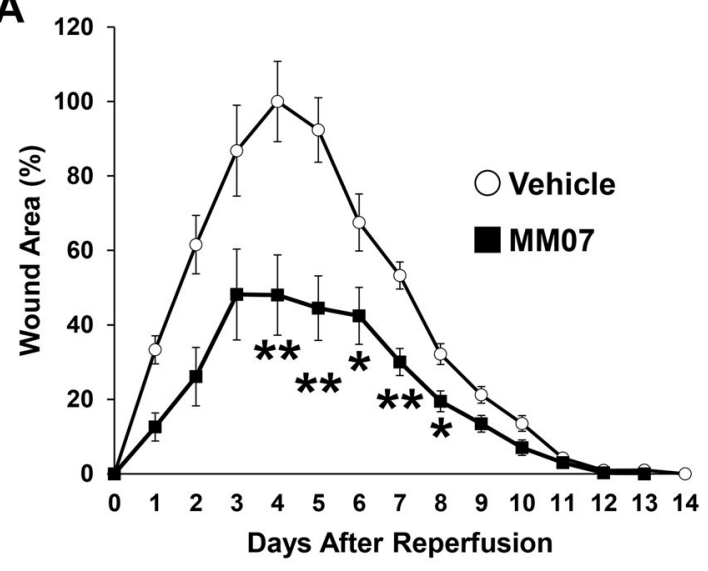

B

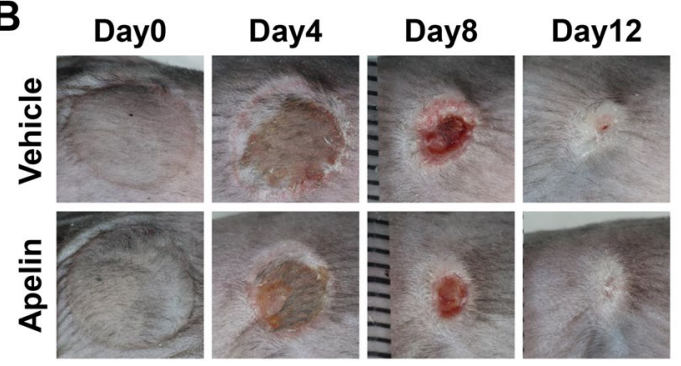

Figure 7. MM07 promoted angiogenesis around I/R area after cutaneous I/R. (A) The size of the wound area after I/R injury in normal C57BL/6 mice treated with subcutaneous injection of MM07 ( $1 \mathrm{ng} / \mathrm{mice})$ or phosphate-buffered saline as a control. The size of the ulcer in control mice at 4 days after reperfusion was assigned a value of $100 \%$ (Control: $\mathrm{n}=8-16$, MM07: $\mathrm{n}=8-16$, for each time point and group). (B) Representative images of the wound after cutaneous $I / R$ in control or apelin mice at $0,4,8$, and 12 days after reperfusion. All values represent mean \pm SEM. $* * P<0.01, * P<0.05$.

\section{Discussion}

At first, we investigated the expression of apelin during cutaneous I/R injury. We found that the expression of apelin was increased by ischemia, and then it was maximized at 4 hours after reperfusion and decreased until 12 hours. After that, it was gradually upregulated again in time dependent manner, suggesting that hypoxia and/ or inflammatory pathways after I/R injury might induce the upregulation of apelin expression in I/R site.

Several studies reported that apelin has protective effect for tissue damage after I/R injury or traumatic injury via suppression of inflammatory cytokines, such as TNF- $\alpha$, IL-1 $\beta$ and IL-6, oxidative stress, autophagy and $\mathrm{N}$-methyl-D-aspartate (NMDA)-induced excitotoxicity ${ }^{16,32,33}$. However, the role of apelin in cutaneous I/R injury is unknown. Therefore, this is the first study to identify the mechanisms of protective regulation in cutaneous $\mathrm{I} / \mathrm{R}$ injury by apelin/APJ signaling.

It has been reported that the number of blood vessels in I/R site were reduced after cutaneous I/R injury, indicating that hypoxia-induced ROS might cause cellular damage and apoptosis ${ }^{7,20}$. In this study, we assessed vascularity in I/R areas and identified that the reductions of $\mathrm{CD} 31^{+}$endothelial cells and $\mathrm{NG}^{+}$pericytes in I/R areas were inhibited by apelin administration, suggesting that apelin might inhibit the damage of blood vessels induced by cutaneous I/R. Apelin has been described to be an activator of angiogenesis and increase blood flow ${ }^{30,34,35}$, suggesting that administration of apelin might increase blood flow and angiogenesis, leading to the inhibition of $\mathrm{I} / \mathrm{R}$ injury-induced vascular damages and hypoxic area in I/R site.

In addition, we demonstrated that enhanced oxidative stress caused by cutaneous I/R was reduced by injection of apelin in OKD48 mice. We also identified that oxidative stress related factors in I/R site were also suppressed in apelin-treated mice. Furthermore, we identified the anti-oxidative effects of apelin through the analysis of the production of ROS in endothelial cells and cellular apoptosis/necrosis in fibroblasts under oxidative stress in vitro. These results suggest that administration of apelin might suppress the oxidative stress and apoptosis via the inhibition of ROS production after cutaneous I/R injury. There are several studies regarding the mechanisms of the reduction of ROS production by apelin. For example, Zeng et al. reported that apelin suppressed ROS production and enhanced superoxide dismutase (SOD) activity in primary cultured myocardial cells after I/R injury ${ }^{17}$. SOD is an enzyme that degrades the ROS generated in cells by oxidative stress. Additionally, it has been reported that apelin-APJ signaling activates the AMP-activated protein kinase (AMPK)/Nrf2 signals, and MAP kinase pathway, resulting in the upregulation of anti-oxidant enzyme, including SOD-1 under oxidative stress condition in adipocytes and neuronal cells ${ }^{36,37}$. These mechanisms may be involved in the suppression of ROS production and oxidative stress by apelin in our study, however further investigation is required. 
Resent study demonstrated that the proinflammatory cytokines derived from inflammatory cells, including neutrophils and macrophages, are key factors in the development of PUs ${ }^{19,22,23}$. In this study, we found the numbers of inflammatory cells were suppressed in apelin-treated mice at day 1 . It has been reported that IL-10, bFGF and TGF- $\beta$ prevent tissue damage after I/R injury ${ }^{38-40}$. We demonstrated that qPCR analysis revealed that apelin administration enhanced the expression of these tissue protective cytokines and growth factors, such as IL-10, bFGF and TGF- $\beta$ in I/R site, however, further studies will be needed to clarify the precise mechanisms.

It has been known that chronic administration of apelin induces $\beta$-arrestin-mediated internalization of APJ and receptor desensitization, suggesting that there is a limitation for the clinical use of apelin ${ }^{31}$. On the other hand, MM07 is cyclic apelin peptide, and preferentially activates G protein responses with low potency in $\beta$-arrestin-mediated receptor internalization ${ }^{30,31}$. Consistent with these findings, it has been reported that intrabrachial infusion of MM07 had greater potential than apelin to increase blood flow in human ${ }^{30}$. Similar to previous results, we identified that inhibitory effect on the development of PUs by MM07 was higher than that by apelin.

Administration of apelin/MM07 suppressed the development of PUs in early phase, however there was no difference in the whole period of wound healing in our study. Since aperin/MM07 has a vasodilatory effect and an oxidative stress inhibitory effect, there is a possibility that it promotes wound healing. However, we considered that there was no difference in the whole period of wound healing because of the strong skin contraction that occurs at the end of wound healing in mice. The limit of this model is that it is highly influenced by the effect of ulcer contraction induced by the fibrosis. This is a so much difference compared by PUs in the humans. Further study will be needed to examine the effect of apelin/MM07 on wound healing.

Taken together, we demonstrate that apelin/APJ signaling suppresses the formation of PUs induced by cutaneous I/R injury by preventing the reduction of blood vessels and the protection of tissue damage through the inhibition of oxidative stress induced by I/R injury. Exogenous apelin or MM07 administration has possible therapeutic potential for cutaneous I/R injury-induced PUs.

\section{Methods}

Animals. All experiments were approved by the Gunma University Animal Care and Experimentation Committee. C57BL/6 mice were purchased from the SLC (Shizuoka, Japan). OKD48 (Keap1-dependent oxidative stress detector, NO-48) mice were kindly provided from Dr. T. Iwawaki (Department of Life Science, Kanazawa Medical University, Ishikawa, Japan). Eight- to 12-week-old mice were used for all experiments. Mice were bred and maintained in the Institute of Experimental Animal Research of Gunma University under specific pathogen-free conditions. Mice were handled in accordance with the animal care guidelines of Gunma University.

Antibodies. Antibodies (Abs) and their sources were as follows: rat anti-mouse CD31 monoclonal Ab (mAb) (MEC13.3; BD Bioscience, San Jose, CA), rabbit anti-mouse NG2 polyclonal Ab (pAb) (Millipore, Billerica, MA), rabbit anti-mouse apelin antibody pAb(Santa Cruz Biotechnology). Alexa 488-, Alexa 568-conjugated secondary Abs were obtained from Invitrogen (Carlsbad, CA). HRP-conjugated goat anti-mouse or anti-rabbit secondary Abs were obtained from Dako (Glostrup, Denmark).

I/R cycles and analysis. The I/R model that has been previously reported was used ${ }^{2,3,19,20,22,23,26}$. Briefly, mice were anesthetized, and hair was shaved and cleaned with $70 \%$ ethanol. The dorsal skin was gently pulled up and trapped between two round ferrite magnetic plates that had a $12-\mathrm{mm}$ diameter $\left(113 \mathrm{~mm}^{2}\right)$ and $5 \mathrm{~mm}$ thick, with an average weight of $2.69 \mathrm{~g}$ and $1180 \mathrm{G}$ magnetic forces (NeoMag Co, Ichikawa, Japan). Epidermis, dermis, subcutaneous fat layer and subcutaneous loose connective tissue layer, but not muscles, were pinched by magnetic plates. This process creates a compressive pressure of $50 \mathrm{mmHg}$ between the two magnets ${ }^{2,3}$. It has been demonstrated that an external pressure of $50 \mathrm{mmHg}$ is sufficient to induce skin necrosis and ulcer by reducing blood flow $80 \%^{3}$. Dorsal skin was trapped between magnetic palates for 12 hours, and then plates were removed. Mice were not immobilized, and not anesthetized during ischemia. All of the mice developed two round ulcers separated by a bridge of normal skin. To assess the effects of apelin ([[Pyr $\left.{ }^{1}\right]$-Apelin-13) (Tocris) or MM07 (cyclo [1-6]CRPRLCHKGPMPF; synthesized by Sigma-Aldrich) ${ }^{30,41}$ on the development of PUs formation and wound healing after cutaneous I/R injury, $10 \mu \mathrm{g}$ of apelin or $1 \mu \mathrm{g}$ of MM07 per $200 \mu \mathrm{l}$ phosphate buffered salts (PBS) or $200 \mu \mathrm{l}$ saline as a control were injected into the dermis in the I/R site just after reperfusion. For analysis, each wound sites were digitally photographed at the indicated time points after wounding, and wound areas were measured on photographs using Image J (version1.48, NIH, Bethesda, MD).

Histological examination and immunofluorescence staining. Immunofluorescence staining of frozen sections and analyses were performed as described previously ${ }^{42}$. Murine skins were removed and $4 \mu \mathrm{m}$ frozen sections were prepared and fixed in 4\% PFA in PBS for 30 minutes. After blocking with 3\% dry milk-PBS supplemented with $5 \%$ normal donkey serum or $5 \%$ normal goat serum for 1 hour at room temperature, sections were stained with Abs of interest followed by Alexa 488-, Alexa 568-conjugated secondary Abs. Sections were counterstained with 4,6-diamidino-2-phenylindole (DAPI) to visualize nuclei, mounted in ProLong Gold antifade reagent (Life Technologies).

Assessment of tissue hypoxia. Hypoxic areas after cutaneous I/R injury in I/R site were detected using the Hypoxyprobe-1 TM Omni kit (HPI, Burlington, MA) as previously described ${ }^{20,43}$. Pimonidazole $\mathrm{HCl}_{\text {was injected }}$ by intraperitoneal $(60 \mathrm{mg} / \mathrm{kg}) 30 \mathrm{~min}$ prior to sacrificing the mice. Murine skins were removed and $4 \mu \mathrm{m}$ frozen sections were prepared and fixed cold acetone (4 degrees Celsius) for $10 \mathrm{~min}$. Sections were incubated overnight at 4 degrees Celsius with rabbit anti-pimonidazole antisera PAb2627 diluted 1:20 in PBS containing 0.1\% bovine serum albumin and $0.1 \%$ Tween 20 . Sections were incubated for $1 \mathrm{~h}$ Alexa 488 -conjugated secondary Abs. Images (6 fields/section) were taken and visualized with a FV10i-DOC confocal laserscanning microscope (Olympus). The positive area was determined by Image (version1.48, NIH, Bethesda, MD) in the field (x600). 
Cell cultures. Mouse embryonic fibroblast cells (NIH3T3) were kindly provided from Dr. S. Torii (Institute for Molecular and Cellular Regulation, Gunma University, Maebashi, Japan). Cells were maintained in Dulbecco's modified Eagle's medium (DMEM) containing 100 units $/ \mathrm{ml}$ penicillin, $100 \mu \mathrm{g} / \mathrm{ml}$ streptomycin and $10 \%$ fetal calf serum (FCS). HUVEC were purchased from ATCC (Manassan, VA). HUVEC were maintained in EBM-2 basal medium (Lonza, Basel, Switzerland) supplemented with EGM-2 Single Quot Kit Suppl. \& Growth Factors (Lonza).

ROS detection assay in vitro. Cellular ROS production were detected as previously ${ }^{26}$. HUVECs $\left(2.5 \times 10^{4}\right.$ cells) were cultured in $\left(100 \mu \mathrm{l} /\right.$ well OptiPlate $^{\mathrm{TM}}-96 \mathrm{~F}$ microplate: Perkin Elmer) incubated at 37 degrees Celsius overnight. Cells were stimulated with $0.75 \mathrm{mM} \mathrm{H}_{2} \mathrm{O}_{2}\left(100 \mu \mathrm{l} /\right.$ well) 2 hours with or without [Pyr ${ }^{1}$-Apelin-13 $(100 \mathrm{nM})$. After that ROS levels was measured with DCFDA Cellular ROS Detection Assay Kit (abcam, Cambridge, UK) according to the manufacturer's protocol with fluorescent microplate measurement.

Apoptosis and necrosis analysis with flow cytometry. Flow cytometric analysis of apoptosis was performed as described previously ${ }^{20,44}$. NIH3T3 cells were incubated in control medium or apelin with or without $\mathrm{H}_{2} \mathrm{O}_{2}(0.75 \mathrm{mM})$ for 24 hours before apoptosis analysis through flow cytometry. Both attached and nonattached cells in the supernatant were corrected. Cells were treated with fluorescein isothiocyanate (FITC)-conjugated Annexin V (BD Bioscience) and 7-amino-actinomycinD (7-AAD), and analyzed with an Attune Focusing Cytometer (Thermo Fisher Scientific, Waltham, MA, USA). Data were processed using FlowJo software (Tree Star Inc., Ashland, OR, USA). Cells that stained positive for Annexin V and negative for 7-AAD were considered to be early apoptotic cells.

Real-time RT-PCR. To analyse the level of mRNA expression at the I/R site by real-time RT-PCR, whole skin samples at the I/R site were used. Total RNA was isolated using an RNeasy Mini Kit (Qiagen, Valencia, CA) and was subjected to reverse transcription using a GoScript Reverse Transcription System for RT-PCR (Promega) according to the manufacturer's instructions. Quantitative RT-PCR was performed with the SYBR system (Applied Biosystems, Foster City, CA) using ABI 7300 real-time PCR instrumentation (Life Technologies) according to the manufacturer's instructions. SYBR probes and primers for Apelin, Nrf2, Trx2, HO-1 and Glyceraldehyde-3-phosphate dehydrogenase (GAPDH) were purchased from Sigma (St. Louis, MO) and Takara Bio Inc. (Otsu, Japan). As an internal control, the levels of GAPDH mRNA were quantified in parallel with the target genes. Normalization and fold-changes were calculated using the comparative Ct method.

Statistics. $\quad P$ values were calculated using the Student's $t$-test (two-sided) or by analysis of one-way ANOVA followed by Bonferroni's post test as appropriate. Error bars represent standard errors of the mean, and numbers of experiments (n) are as indicated.

Received: 15 August 2019; Accepted: 15 January 2020;

Published online: 28 January 2020

\section{References}

1. Salcido, R. et al. Histopathology of pressure ulcers as a result of sequential computer-controlled pressure sessions in a fuzzy rat model. Advances in wound care: the journal for prevention and healing 7, 23-4, 26, 28 passim (1994).

2. Peirce, S. M., Skalak, T. C. \& Rodeheaver, G. T. Ischemia-reperfusion injury in chronic pressure ulcer formation: a skin model in the rat. Wound repair and regeneration: official publication of the Wound Healing Society [and] the European Tissue Repair Society $\mathbf{8}$, 68-76 (2000).

3. Stadler, I., Zhang, R. Y., Oskoui, P., Whittaker, M. S. \& Lanzafame, R. J. Development of a simple, noninvasive, clinically relevant model of pressure ulcers in the mouse. Journal of Investigative Surgery 17, 221-227 (2004).

4. Pretto, E. A. Reperfusion injury of the liver. Transplantation proceedings 23, 1912-4 (1991)

5. Woolfson, R. G., Millar, C. G. \& Neild, G. H. Ischaemia and reperfusion injury in the kidney: current status and future direction. Nephrology, dialysis, transplantation: official publication of the European Dialysis and Transplant Association - European Renal Association 9, 1529-31 (1994).

6. Carroll, W. R. \& Esclamado, R. M. Ischemia/reperfusion injury in microvascular surgery. Head \& neck 22, 700-13 (2000).

7. Kasuya, A., Sakabe, J. I. \& Tokura, Y. Potential application of in vivo imaging of impaired lymphatic duct to evaluate the severity of pressure ulcer in mouse model. Scientific Reports 4, 4173 (2014).

8. Tatemoto, K. et al. Isolation and characterization of a novel endogenous peptide ligand for the human APJ receptor. Biochemical and Biophysical Research Communications 251, 471-476 (1998).

9. Lee, D. L. et al. Characterization of apelin, the ligand for the APJ receptor. Journal of Neurochemistry 74, 34-41 (2000).

10. Berry, M. F. et al. Apelin has in vivo inotropic effects on normal and failing hearts. Circulation 110, 187-193 (2004).

11. Kidoya, H. \& Takakura, N. Biology of the apelin-APJ axis in vascular formation. Journal of Biochemistry 152, 125-131 (2012).

12. Kidoya, H. et al. APJ Regulates Parallel Alignment of Arteries and Veins in the Skin. Developmental Cell 33, 247-259 (2015).

13. Boucher, J. et al. Apelin, a newly identified adipokine up-regulated by insulin and obesity. Endocrinology 146, 1764-1771 (2005).

14. Day, R. T., Cavaglieri, R. C. \& Feliers, D. Apelin retards the progression of diabetic nephropathy. American Journal of PhysiologyRenal Physiology 304, F788-F800 (2013).

15. Nishida, M. et al. The Role of Apelin on the Alleviative Effect of Angiotensin Receptor Blocker in Unilateral Ureteral ObstructionInduced Renal Fibrosis. Nephron Extra 2, 39-47 (2012).

16. Bircan, B., Çakır, M., Kırbağ, S. \& Gül, H. F. Effect of apelin hormone on renal ischemia/reperfusion induced oxidative damage in rats. Renal Failure 38, 1122-1128 (2016).

17. Zeng, X. J. et al. Apelin protects heart against ischemia/reperfusion injury in rat. Peptides 30, 1144-1152 (2009).

18. Yang, Y. et al. Apelin-13 protects the brain against ischemia/reperfusion injury through activating PI3K/Akt and ERK1/2 signaling pathways. Neuroscience Letters 568, 44-49 (2014).

19. Saito, Y. et al. The loss of MCP-1 attenuates cutaneous ischemia-reperfusion injury in a mouse model of pressure ulcer. Journal of Investigative Dermatology 128, 1838-1851 (2008).

20. Motegi, S. et al. Protective effect of mesenchymal stem cells on the pressure ulcer formation by the regulation of oxidative and endoplasmic reticulum stress. Scientific Reports 7, 17186 (2017).

21. Sendoel, A. \& Hengartner, M. O. Apoptotic Cell Death Under Hypoxia. Physiology 29, 168-176 (2014) 
22. Uchiyama, A. et al. Protective Effect of MFG-E8 after Cutaneous Ischemia-Reperfusion Injury. Journal of Investigative Dermatology 135, 1157-1165 (2015).

23. Uchiyama, A. et al. Topical betamethasone butyrate propionate exacerbates pressure ulcers after cutaneous ischemia-reperfusion injury. Experimental Dermatology 25, 678-683 (2016).

24. Oikawa, D., Akai, R., Tokuda, M. \& Iwawaki, T. A transgenic mouse model for monitoring oxidative stress. Scientific Reports 2, 229 (2012).

25. Takahashi, S. et al. An Nrf2/Small Maf Heterodimer Mediates the Induction of Phase II Detoxifying Enzyme Genes through Antioxidant Response Elements. Biochemical and Biophysical Research Communications 236, 313-322 (2002).

26. Sekiguchi, A. et al. Botulinum toxin B suppresses the pressure ulcer formation in cutaneous ischemia-reperfusion injury mouse model: Possible regulation of oxidative and endoplasmic reticulum stress. Journal of Dermatological Science 90, 144-153 (2018).

27. Sun, C.-K. et al. Melatonin treatment enhances therapeutic effects of exosomes against acute liver ischemia-reperfusion injury. American journal of translational research 9, 1543-1560 (2017).

28. Takagi, T. et al. Temporal activation of Nrf2 in the penumbra and Nrf2 activator-mediated neuroprotection in ischemia-reperfusion injury. Free Radical Biology and Medicine 72, 124-133 (2014).

29. Sun, N. et al. GluR6-FasL-Trx2 mediates denitrosylation and activation of procaspase-3 in cerebral ischemia/reperfusion in rats. Cell death \& disease 4, e771 (2013).

30. Brame, A. L. et al. Design, characterization, and first-in-human study of the vascular actions of a novel biased apelin receptor agonist. Hypertension 65, 834-840 (2015).

31. Yang, P., Maguire, J. J. \& Davenport, A. P. Apelin, Elabela/Toddler, and biased agonists as novel therapeutic agents in the cardiovascular system. Trends in Pharmacological Sciences 36, 560-567 (2015).

32. Bao, H. J., Zhang, L., Han, W. C. \& Dai, D. K. Apelin-13 Attenuates Traumatic Brain Injury-Induced Damage by Suppressing Autophagy. Neurochemical Research 40, 89-97 (2014).

33. Zeng, X. J., Yu, S. P., Zhang, L. \& Wei, L. Neuroprotective effect of the endogenous neural peptide apelin in cultured mouse cortical neurons. Experimental Cell Research 316, 1773-1783 (2010).

34. Sorli, S. C., Le Gonidec, S., Knibiehler, B. \& Audigier, Y. Apelin is a potent activator of tumour neoangiogenesis. Oncogene 26, 7692-7699 (2007).

35. Kidoya, H. et al. Spatial and temporal role of the apelin/APJ system in the caliber size regulation of blood vessels during angiogenesis. EMBO Journal 27, 522-534 (2008).

36. Duan, J. et al. Neuroprotective effect of Apelin 13 on ischemic stroke by activating AMPK/GSK-3//Nrf2 signaling. Journal of Neuroinflammation 16, 24 (2019).

37. Than, A. et al. Apelin attenuates Oxidative stress in human Adipocytes. Journal of Biological Chemistry 289, 3763-3774 (2014).

38. Sakai, K. et al. Protective effect and mechanism of IL-10 on renal ischemia-reperfusion injury. Laboratory Investigation 99, 671-683 (2019).

39. Xiao, J. et al. Cardiac protection by basic fibroblast growth factor from ischemia/reperfusion-induced injury in diabetic rats. Biological and Pharmaceutical Bulletin 33, 444-449 (2010).

40. Lefer, A. M., Ma, X. L., Weyrich, A. S. \& Scalia, R. Mechanism of the cardioprotective effect of transforming growth factor $\beta 1$ in feline myocardial ischemia and reperfusion. Proceedings of the National Academy of Sciences of the United States of America 90, 1018-1022 (1993).

41. Yokoyama, Y. et al. Inhibitory Regulation of Skin Fibrosis in Systemic Sclerosis by Apelin/APJ Signaling. Arthritis and Rheumatology 70, 1661-1672 (2018)

42. Motegi, S. et al. Pericyte-derived MFG-E8 regulates pathologic angiogenesis. Arteriosclerosis, Thrombosis, and Vascular Biology 31, 2024-2034 (2011).

43. Uchiyama, A. et al. Protective effect of botulinum toxin A after cutaneous ischemia-reperfusion injury. Scientific Reports 5, 9072 (2015).

44. Motegi, S. et al. Increased susceptibility to oxidative stress- and ultraviolet A-induced apoptosis in fibroblasts in atypical progeroid syndrome/atypical Werner syndrome with LMNA mutation. Experimental Dermatology 25, 20-27 (2016).

\section{Acknowledgements}

This work was partly supported by a Lidia O'Leary Memorial Pias Dermatological Foundation (to S.M.).

\section{Author contributions}

S.Y. and S.M. involved in all the process of planning and implementing the experiment, interpretation of data, and writing the manuscripts. A.S., A.U., C.F., Y.I., Y.Y., S.O., R.T., M.H., R.A. and T.I. involved in the process of implementing the experiment. O.I. involved in the process of planning the experiment, interpretation of data, and writing the manuscripts. All authors reviewed the manuscript.

\section{Competing interests}

The authors declare no competing interests.

\section{Additional information}

Correspondence and requests for materials should be addressed to S.-i.M.

Reprints and permissions information is available at www.nature.com/reprints.

Publisher's note Springer Nature remains neutral with regard to jurisdictional claims in published maps and institutional affiliations.

Open Access This article is licensed under a Creative Commons Attribution 4.0 International License, which permits use, sharing, adaptation, distribution and reproduction in any medium or format, as long as you give appropriate credit to the original author(s) and the source, provide a link to the Creative Commons license, and indicate if changes were made. The images or other third party material in this article are included in the article's Creative Commons license, unless indicated otherwise in a credit line to the material. If material is not included in the article's Creative Commons license and your intended use is not permitted by statutory regulation or exceeds the permitted use, you will need to obtain permission directly from the copyright holder. To view a copy of this license, visit http://creativecommons.org/licenses/by/4.0/.

(C) The Author(s) 2020 